\title{
Role of Positron Emission Tomography with 2-Deoxy-2-[fluorine-18] fluoro-D-glucose Integrated with Computed Tomography in the Evaluation of Hepatic Metabolic Activity due to Steatosis in Lymphoma Patients and its Impact on Deauville Score
}

\author{
Marwa Adel*, Ashraf Fawzy, Jehan Younes, Shaimaa EIRasad \\ Department of Oncology and Nuclear Medicine, Cairo University, Kasr-Alainy Center of Clinical Oncology and Nuclear Medicine, \\ Kasr-El-Ainy Hospital, Egypt
}

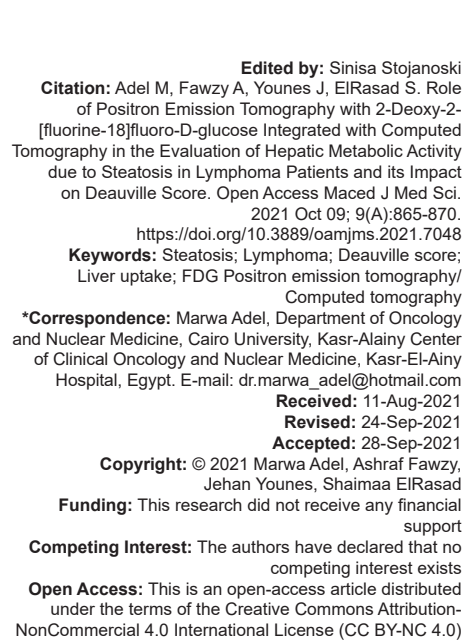

Background

Lymphomas are the most common primary hematopoietic malignancy [1]. They are heterogeneous group of lymphoid malignancies, which can be broadly divided into non-Hodgkin lymphomas (NHLs) and Hodgkin lymphomas (HLs) that display different patterns of biological behavior and response to treatment [2]. Positron emission tomography (PET)computed tomography (CT) with 18F-FDG is a standard staging procedure for most lymphoma subtypes. Performed before and after therapy for HL and aggressive NHL, 18F-FDG PET results have a high prognostic value and correlate with survival [3]. Fatty liver is the leading cause of liver enzyme abnormalities in the developed countries [4]. Patients with fatty liver are at risk of metabolic comorbidities. This disease must not be ignored specially in our country; Egypt is considered a highest endemic area for the prevalence of hepatitis $\mathrm{C}$ virus (HCV) infection. Liver steatosis or steatohepatitis represents a comorbid condition that accelerates progression of chronicity; morbidity and mortality among patients with chronic HCV infection. Fatty liver can also be a feature of drug-induced liver injury and was described in patients treated with methotrexate, amiodarone, antiretrovirals, and estrogen receptor modulators, such as tamoxifen [4]. Fatty liver development was also reported as a consequence of cancer chemotherapy, especially for colorectal cancer with treatments containing 5-fluorouracil or irinotecan [4]. The diagnosis of non-alcoholic fatty liver disease (NAFLD) is evaluated by several methods, including liver biopsy, as well as non-invasive radiological modalities, such as CT (discussed later), magnetic resonance imaging, magnetic resonance spectroscopy, and ultrasonography [5].

Standardized uptake value (SUV) is increasingly used in clinical studies in addition to visual assessments. SUV is a measurement of the uptake 
in a tumor normalized on the basis of a distribution volume. Most of the published literature relates to SUV (normalized to body weight) measurements. SUV normalized to lean body mass (LBM) is referred to as SUL and is a recommended quantitative measure of FDG uptake. The use of SUL is preferred for response assessment studies when large changes in body weight may occur during the course of the treatment [6].

\section{Methods}

This prospective study was performed in Kasr-Alainy Center of Clinical Oncology and Nuclear Medicine (NEMROCK) after being approved by the ethical committee. A total of 77 cases pathologically proven to have lymphoma (Hodgkin and NHL) presented to the NEMROCK between April 2019 and December 2020. The patients were subjected to whole body PET with 2-deoxy-2-[fluorine-18]fluoro-D-glucose integrated with CT (18F-FDG PET/CT) as a baseline staging before therapy, in the middle of their $1^{\text {st }}$ line of chemotherapy interim 18F-FDG PET/CT and end of treatment and/or follow-up (EOT/FU) were performed for the assessment of response.

The medical history of these patients was checked and only patients meeting inclusion criteria and for whom international guidelines for PET tumor imaging had been fulfilled which were included in the study. For each patient, age, sex, initial Ann Arbor staging, history of diabetes, virology (hepatitis B virus [HBV] and HCV), and liver dysfunction were recorded. We excluded pregnant ladies and patients who had active second malignancy.

Patients were instructed for preparation according to the EANM procedure guidelines for 18F-FDG PET/CT tumor imaging: Version 2.0 [6]. Briefly, patients were required to fast for $6 \mathrm{~h}$ and asked to void before the examination. The patients were instructed to stay calm and avoid walking or any exercise before and after injection of 18F-FDG to prevent physiologic muscle FDG uptake. Blood glucose level measured before injection, and fasting levels were $<200 \mathrm{mg} / \mathrm{dL}$ (70-170 mg/dL in our cohort). Warm environment was available before injection to avoid brown fat uptake.

A dose of 3.7-5.2 MBq/Kg of 18F-FDG was injected through a hand or antecubital vein. The injection was performed 45-90 min before the start of PET/CT acquisition. For opacification of bowel loops, $400-600 \mathrm{ml}$ of contrast material diluted with water and swallowed $1 \mathrm{~h}$ before PET/CT imaging.

The scanner used in NEMROCK was Ingenuity TF 64 (Philips Healthcare, Cleveland, $\mathrm{OH}$, USA) a PET/CT scanner combining a modular,
LYSO-based PET component with a 64-channel CT component. The CT was based on the Ingenuity $\mathrm{CT}$ (Philips Healthcare).

Acquisition and reconstruction protocol were previously described [6]. Briefly, a low-dose non-contrast CT scan was performed first, followed by a whole-body PET acquisition then a whole-body contrast-enhanced CT scan. Low-dose CT was acquired in a helical mode, using $120 \mathrm{kV}, 60 \mathrm{mAs}$, and a $512 \times 512$ matrix size, acquiring a field of view (FOV) of $700 \mathrm{~mm}$. This CT scan was used for attenuation correction.

PET scan was acquired in a three-dimensional mode over the same anatomical regions starting from the skull vertex to the level of the mid-thigh. The acquisition time was 2 min per bed position, in nine bed positions. Reconstructed slice thickness was $5 \mathrm{~mm}$.

Immediately after completing PET acquisition, a diagnostic CT with contrast was acquired using $120 \mathrm{kV}, 300 \mathrm{mAs}$, and a $512 \times 512$ matrix size. The acquired FOV was $500 \mathrm{~mm}$ using dose automatic modulation in the $\mathbf{Z}$ direction. Non-ionic contrast media was IV injected using automatic injector at a rate of $4 \mathrm{ml} / \mathrm{s}$, in a dose of 1-2 ml/kg (maximum <150 ml). Slice thickness was $1.0 \mathrm{~mm}$.

The whole study took about 20-30 $\mathrm{min}$. Raw data were reconstructed using a standard manufacturer's iterative algorithm. Axial PET and CT images obtained and then reformatted into sagittal and coronal images to allow easier image interpretation.

\section{PET/CT and CE-CT interpretation}

\section{PET/CT analysis}

For each PET-CT exam, liver maximum SUV (SUVmax), lean body SUV max (SULmax), and liver mean Hounsfield units (HU) were measured using an automatic $3 \mathrm{~cm}$ diameter volume of interest (VOI) set in the right liver lobe, avoiding liver lesions in the case of focal liver involvement. Spleen mean HU was also recorded using a $2 \mathrm{~cm}$ diameter VOI. Several cutoff values were used to define steatosis: Mean liver HU $\leq 42$, ratio between liver and spleen mean $\mathrm{HU}$ values $(\mathrm{CTL} / \mathrm{S}) \leq 0.8$, and difference between liver and spleen mean HU values (CTL-S) $\leq-9$ [7]. SUVmax and SULmax in the mediastinum were measured in an automatically placed $1 \mathrm{~cm}$ diameter and $2 \mathrm{~cm}$ height cylinder in the descending thoracic aorta. In baseline examinations and in case of remaining lesions in interim and EOT/ FU examinations, the most intense target lesion was located by upscaling the base of the look up table on the 3D MIP view. SUVmax and SULmax were computed as follows:

SUVmax=Measured activity $\mathrm{x}$ body weight $(\mathrm{kg})$ /injected dose (MBq)

SULmax=measured activity $x$ LBM $(\mathrm{kg}) /$ injected dose (MBq). 
The Deauville 5-point scale (DS) was used to evaluate response for each interim and post-treatment $\mathrm{PET} / \mathrm{CT}$ examination

DS1 $=$ No uptake

DS2 = Uptake $\leq$ Mediastinum

DS3 $=$ Uptake $>$ Mediastinum but $\leq$ Liver

DS4 =Moderately increased uptake compared to the liver

DS5= Markedly increased uptake compared to the liver (defined as 2 times liver) and/or new lesions

\section{Statistical analysis}

Data were statistically described in terms of mean \pm standard deviation ( $\pm \mathrm{SD})$, median, range, and $I Q R$, or frequencies (number of cases) and percentages when appropriate. Numerical data were tested for the normal assumption using Kolmogorov-Smirnov test. Comparison of numerical variables between the study groups was done using Student's t-test for independent samples in comparing two groups of normally distributed data and Mann-Whitney U-test for independent samples for comparing not normal data. Comparison between more than 2 groups was done using Kruskal-Wallis test. For comparing categorical data, Chi-square $\left(\chi^{2}\right)$ test was performed. Exact test was used instead when the expected frequency is $<5$. Correlation between various variables was done using Pearson moment correlation equation for linear relation of normally distributed variables and Spearman rank correlation equation for non-normal variables/non-linear monotonic relation. Correction of liver SULmax values was done according to the method described by Solomon et al., 2017. Twosided $p<0.05$ was considered statistically significant. All statistical calculations were done using computer program IBM SPSS (Statistical Package for the Social Sciences; IBM Corp, Armonk, NY, USA) release 22 for Microsoft Windows.

\section{Results}

A total of 77 cases were included, the mean age $=39.5+13,49$ patients were female $(63.6 \%)$, 28 patients were male $(36.4 \%), 41$ patients $(53.2 \%)$ diagnosed as $\mathrm{NHL}$ while 36 patients $(46.8 \%)$ diagnosed as HL; Ann Arbor Stage I, II, III, or IV was found in $6(7.8 \%), 25(32.5 \%), 25(32.5 \%)$, and 21 patients (27.3\%), respectively, as. All 77 patients received first line of treatment after baseline PET/CT; 36 patients received ABVD (46.8\%), 39 patients received $\mathrm{R}-\mathrm{CHOP}$ $(50.7 \%)$, and 2 patients received REPOCH $(2.5 \%)$. Nine patients out of 68 patients (who did interim PET/ $\mathrm{CT}$ ) received second line of treatment after interim PET/CT; DHAP $(2 / 9,22.2 \%)$, ESHAP $(6 / 9,66.6 \%)$, and lenalidomide $(1 / 9,11.1 \%)$ (Table 1$)$.
Table 1: Population characteristics

\begin{tabular}{|c|c|c|}
\hline Characteristics & No & $\%$ \\
\hline \multicolumn{3}{|l|}{ Age, years } \\
\hline Mean \pm SD & $39.5 \pm 13$ & \\
\hline \multicolumn{3}{|l|}{ Gender } \\
\hline Female & 49 & 63.6 \\
\hline Male & 28 & 36.4 \\
\hline \multicolumn{3}{|l|}{ Ann Arbor stage } \\
\hline 1 & 6 & 7.8 \\
\hline II & 25 & 32.5 \\
\hline III & 25 & 32.5 \\
\hline IV & 21 & 27.3 \\
\hline \multicolumn{3}{|l|}{ Pathology } \\
\hline NHL & 41 & 53.2 \\
\hline $\mathrm{HL}$ & 36 & 46.8 \\
\hline \multicolumn{3}{|l|}{ First line of CTH } \\
\hline ABVD & 36 & 46.8 \\
\hline $\mathrm{R}-\mathrm{CHOP}$ & 39 & 50.7 \\
\hline REPOCH & 2 & 2.5 \\
\hline \multicolumn{3}{|c|}{ Second line of CTH } \\
\hline DHAP & 2 & 22.2 \\
\hline ESHAP & 6 & 66.6 \\
\hline Lenalidomide & 1 & 11.1 \\
\hline \multicolumn{3}{|l|}{ HCV } \\
\hline Positive & 5 & 6.5 \\
\hline Negative & 72 & 93.5 \\
\hline \multicolumn{3}{|l|}{ HBV } \\
\hline Positive & 3 & 3.9 \\
\hline Negative & 74 & 96.1 \\
\hline \multicolumn{3}{|c|}{ Baseline liver functions } \\
\hline AST & $19(12-22)$ & \\
\hline ALT & $24(21.5-39.5)$ & \\
\hline \multicolumn{3}{|c|}{ Liver functions in FU } \\
\hline AST & $20(15-25)$ & \\
\hline ALT & $30(24-40)$ & \\
\hline
\end{tabular}

ABVD: Adriamycin, bleomycin, vinblastine, dacarbazine, REPOCH: Rituximab, Etoposide, prednisone, vincristine, cyclophosphamide, doxorubicin, R-CHOP: Rituximab, cyclophosphamide, hydroxydaunomycin, oncovin, prednisolone, DHAP: (D)examethasone, (H)igh-dose (A)ra-C - cytarabine, (P)latinol (cisplatin), ESHAP: Etoposide, methyl-prednisolone, cytarabine (Ara-C), cisplatin (platinum)

\section{Steatosis in baseline, interim, and EOT PET/CT}

There was no significant difference between liver density (steatosis and non-steatosis) in baseline $\mathrm{PET} / \mathrm{CT}$ versus interim PET/CT with $p=0.3$ and in interim PET/CT versus EOT PET/CT with $p=0.6$ (Tables 2 and 3 ).

Table 2: Cross-tabulation between baseline and interim PET/CT

\begin{tabular}{|c|c|c|c|c|}
\hline \multirow{2}{*}{\multicolumn{2}{|c|}{ Characteristics }} & \multicolumn{2}{|l|}{ Interim } & \multirow[t]{2}{*}{$p$ value } \\
\hline & & Steatosis & Non-steatosis & \\
\hline \multirow[t]{4}{*}{ Baseline } & Steatosis & 6 & 3 & 0.3 \\
\hline & & $8.7 \%$ & $4.3 \%$ & \\
\hline & Non-steatosis & 7 & 53 & \\
\hline & & $10.1 \%$ & $76.8 \%$ & \\
\hline \multirow{2}{*}{\multicolumn{2}{|c|}{ Total $=69(100 \%)$}} & 13 & 56 & \\
\hline & & $18.8 \%$ & $81.2 \%$ & \\
\hline
\end{tabular}
due to their small sample size.

In this comparison, cases that lost interim or EOT PET/CT were excluded, therefore, 68 patients were included in comparison between baseline and interim PET/CT and 23 patients were included in comparison between interim and EOT PET/CT (Tables 2 and 3).

Table 3: Cross-tabulation between interim and EOT/FU3

\begin{tabular}{lllll}
\hline Characteristics & & \multicolumn{2}{l}{ Interim } & p value \\
\cline { 3 - 4 } & & Steatosis & Non-steatosis & \\
\hline EOT/FU & Steatosis & 2 & 1 & 0.6 \\
& & $8.7 \%$ & $4.3 \%$ & \\
& Non-steatosis & 3 & 17 & \\
Total $=23(100 \%)$ & $13 \%$ & $73.9 \%$ & \\
& 5 & 18 & \\
\hline
\end{tabular}




\section{$P E T / C T$}

\section{Development of steatosis in interim}

Seven patients $(10.1 \%)$ developed steatosis in interim PET/CT; six out of seven patients who developed hepatic steatosis in interim PET/CT had BMI $>30 \mathrm{~kg} / \mathrm{m}^{2}$ (three patients received $\mathrm{RCHOP}$ while others received ABVD), however, one patient out of seven patients who developed steatosis in interim PET/CT had BMI $<30 \mathrm{~kg} / \mathrm{m}^{2}$ diagnosed as NHL and received 3 cycles of $\mathrm{R}-\mathrm{CHOP}$ as shown in Table 2.

\section{$P E T / C T$}

\section{Disappearance of steatosis in interim}

Three patients had a disappearance of steatosis interim PET/CT, two patients of them were male with $\mathrm{BMI}<30 \mathrm{~kg} / \mathrm{m}^{2}$, however, one patient was female with BMI >30 kg/m² as shown in Table 2 .

\section{Development of steatosis in EOT PET/CT}

One female patient developed steatosis in EOT diagnosed as HL received 6 cycles of ABVD and had BMI $23.5 \mathrm{~kg} / \mathrm{m}^{2}$ (Table 3).

Disappearance of steatosis was recorded in three patients (two male patients and one female patient) in EOT with BMI $<30 \mathrm{~kg} / \mathrm{m}^{2}$ (Table 3).

Table 4: Correlation between BGL, HBV, HCV, BMI, and steatosis and each of liver SUVmax and SULmax in interim

\begin{tabular}{|c|c|c|c|c|}
\hline & & & Liver SUVmax & Liver SULmax \\
\hline Spearman's rho & BMI & Correlation coefficient & 0.573 & -0.116 \\
\hline \multirow[t]{7}{*}{$\mathrm{n}=69$ patients } & & $p$ value & 0.001 & 0.344 \\
\hline & Steatosis & Correlation coefficient & 0.087 & -0.116 \\
\hline & & $\mathrm{p}$ value & 0.478 & 0.344 \\
\hline & HBV & Correlation coefficient & -0.079 & -0.270 \\
\hline & & $p$ value & 0.520 & 0.025 \\
\hline & $\mathrm{HCV}$ & $\begin{array}{l}\text { Correlation coefficient } \\
\text { p value }\end{array}$ & $\begin{array}{l}-0.231 \\
0.056\end{array}$ & $\begin{array}{l}-0.011 \\
0.929\end{array}$ \\
\hline & BGL & Correlation coefficient & $\begin{array}{l}-0.007 \\
0.955\end{array}$ & $\begin{array}{l}-0.033 \\
0.789\end{array}$ \\
\hline
\end{tabular}

\section{Correlations of liver uptake on interim and} EOT PET scan (77 cases)

1-Correlation of steatosis, blood glucose level, BMI, HBV, and HCV with each of SUVmax and SULmax in interim and EOT PET/CT

Median/range of liver SUVmax and SULmax in interim PET/CT was 2.3 (1.9-2.9) and 1.6 (1.3-2), respectively.

Median/range of liver SUVmax and SULmax in EOT PET/CT was 2.6 (2.2-3) and 1.7 (1.3-2.8), respectively.

Median/range of BMI in interim and EOT PET/CT was 28.3 (22.2-32.4) and 27.5 (24.4-32.8), respectively.

There was a positive correlation between liver SUVmax values with BMI in both interim and EoT groups with an $r$ value equal to $0.5(p<0.001)$ and 0.67 $(p<0.001)$, respectively.

Table 5: Correlation between BGL, HBV, HCV, BMI, and steatosis and each of liver SUVmax and SULmax in EOT/FU

\begin{tabular}{lllll}
\hline & & Liver SUVmax & Liver SULmax \\
\hline $\begin{array}{llll}\text { Spearman's rho } \\
\mathrm{n}=31 \text { patients }\end{array}$ & BMI & Correlation coefficient & 0.672 & -0.127 \\
& & p value & 0.001 & 0.521 \\
& Steatosis & Correlation coefficient & -0.065 & -0.114 \\
& p value & 0.729 & 0.557 \\
& HBV & Correlation coefficient & 0.041 & -0.204 \\
& HCV & p value & 0.827 & 0.289 \\
& Correlation coefficient & 0.098 & 0.081 \\
& BGL & p value & 0.600 & 0.675 \\
& Correlation coefficient & 0.154 & 0.086 \\
& p value & 0.407 & 0.659 \\
\hline
\end{tabular}

There was no correlation between steatosis, blood glucose level, HCV, HBV, and each of liver SUVmax and SULmax in both interim and EOT PET/ CT (Tables 4 and 5).

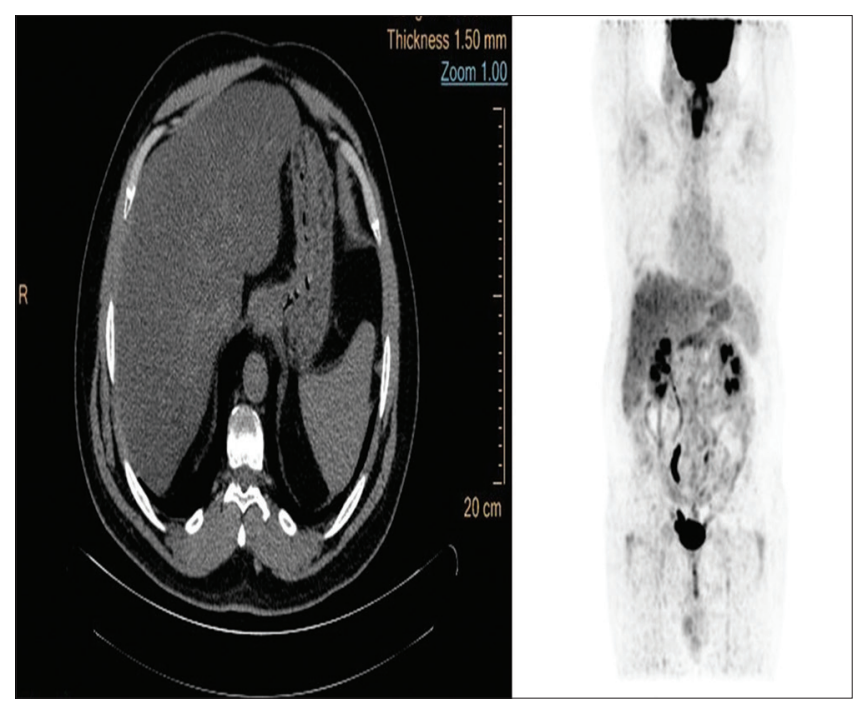

Figure 1: Representative example of steatotic patient. Maximal intensity projection and axial computed tomography (CT) image for a 45-year-old male patient addressed for interim positron emission tomography/CT of Hodgkin lymphoma scored Deauville score 2. Body mass index (BMI) was increasing throughout follow-up. BMI; initially was $29.8 \mathrm{~kg} / \mathrm{m}^{2}$, BMI at interim and EOT was 34.3 and $33.6 \mathrm{~kg} / \mathrm{m}^{2}$, respectively

Representative example of steatotic patient is shown in Figure 2 and representative example of nonsteatotic patient is shown in Figure 2.

\section{Discussion}

Lymphoma broadly divided into Hodgkin's and non-Hodgkin's lymphoma, and it accounts for one of the most common malignant diseases in the general population [8]. Steatosis or NAFLD affects 10-24\% of the general population in different countries [6]. Drug-induced fatty liver was described in patients treated with chemotherapy. It is difficult to determine prevalence of chemotherapy induced fatty liver due to heterogeneity in treatment regimens [4]. Our aim 
is to evaluate the prevalence of hepatic steatosis in patients with lymphoma and its impact on Deauville score (DS).

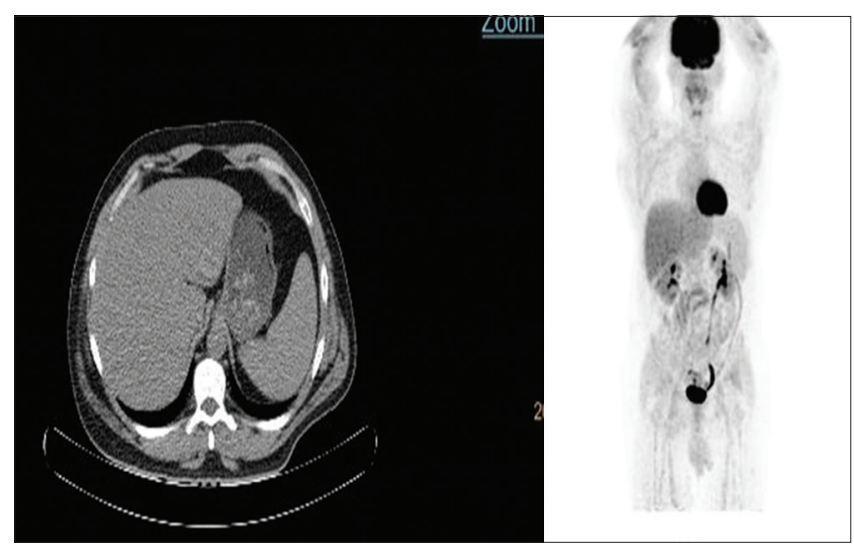

Figure 2: Representative example of non-steatotic patient. Maximal intensity projection and axial computed tomography (CT) image for a 42-year-old male patient addressed for interim positron emission tomography (PET)/CT of HL scored DS 1. Body mass index (BMI) was mildly increasing throughout follow-up. BMI was 36.2 and $37 \mathrm{~kg} /$ $\mathrm{m}^{2}$ during baseline and interim PET/CT examinations, respectively.

In our study, the prevalence of steatosis in baseline $(10 / 77,12.9 \%)$, interim $(13 / 69,18.8 \%)$, and EOT/FU (4/31, 12.9\%); this is more than prevalence of steatosis in general population which is in contrary to Salomon et al. [2]. In our study, development of hepatic steatosis was documented in eight patients during their course of treatment (seven patients in interim PET/CT and one patient in EOT PET/CT). In comparison to Salomon et al. [6], one patient developed steatosis during his course of treatment, he had BMI $>30$. However, in our study, there was no statistically significant difference in development of hepatic steatosis throughout different time of PET/CT examinations. Six patients out of seven patients had $\mathrm{BMI}>30 \mathrm{~kg} / \mathrm{m}^{2}$ in interim PET/CT, however, other two patients had BMI $<30 \mathrm{~kg} / \mathrm{m}^{2}$. Unfortunately, other factors influencing fatty liver were limited in our study as certain drug intake, lipid profile, history of dyslipidemia, or metabolic risk factors. Similar to Salomon et al. [6], we found that hepatic steatosis was not apparently to the timecourse of treatment and therefore does not explain the variability of liver 18F-FDG uptake previously observed in patients.

According to liver metabolic activity in our study, there was no significant relation regarding hepatic steatosis, HBV, HCV, blood glucose level, and different lines of chemotherapy with liver metabolic activity either using liver SUVmax or SULmax in interim or EOT $\mathrm{PET} / \mathrm{CT}$ studies. This is in concordance with Salomon et al. [6], Ben Yakov et al. [3], and Lin et al. [9], [10], but we disagreed with Salomon et al. [6] and Keramida et al. [11], regarding correlation between liver SUVmax in EOT PET/CT and liver SULmax in interim and EOT PET/CT with steatosis, they found that liver SULmax and SUVmax were significantly lower in steatotic patients.
Using 5-point scales of DS as visual and semi-quantitative analysis in response assessment in Iymphoma patients, DS1-3 versus D4-5 is used to discriminate between responders and non-responders, respectively. This score has been shown to have a prognostic value early in the course of treatment and/or at the end of the treatment. SUV normalized by body weight is affected by amount of body fat. SUV calculated/ normalized by LBM (LBM, fat-free body mass) (SUVLBM or SUL) instead of total weight is recommended to provide more accurate SUV results [12].

In light of above-mentioned reasons, we are in accordance with Salomon et al. and Sarikaya et al. [6], [12] that higher BMI is associated with high liver SUVmax. However, no statistical correlation was found between BMI and liver SULmax. We used liver SULmax values instead of the recommended SUVmax values for the determination of DS. In our study, SULmax values gave the same DS as SUVmax values. These results suggest that either SUVmax or SULmax can be used to score patients with relatively consistent results.

\section{Conclusion}

\section{There are limitations in our study}

First, we need larger and more homogeneous studies of patients initially scored DS4. Second, limited number of patients especially steatotic for better assessment of liver uptake in steatotic patients and using of liver correction methods. Third, other factors interfering with fatty liver as certain drug intake, lipid profile, history of dyslipidemia, or metabolic risk factors.

We concluded that irrespective to hepatic steatosis, virology, blood glucose level, and BMI was significantly correlated with liver SUVmax. In contrary, liver SULmax had no significant correlation with BMI. This confirms fact that SUV normalized by body weight is affected by amount of body fat. SUV calculated/normalized by LBM (LBM, fat-free body mass) (SUVLBM or SUL) instead of total weight is recommended to provide more accurate SUV results. Using SULmax has the advantage of giving the opportunity to reveal and potentially take into account parameters other than BMI that could influence the liver uptake. Liver SULmax values gave the same DS as SUVmax values. These results suggest that either SUVmax or SULmax can be used to score patients with relatively consistent results. According to the EANM procedure guidelines for tumor imaging, the use of SUL is preferred for response assessment studies when large changes in body weight may occur during the course of the treatment. 


\section{References}

1. Okada M, Sato N, Ishii K, Matsumura K, Hosono M, Murakami T. FDG PET/CT versus CT, MR imaging, and $67 \mathrm{Ga}$ scintigraphy in the posttherapy evaluation of malignant lymphoma. Radiographics. 2010;30(4):939-57. https://doi.org/10.1148/ rg.304095150

PMid:20631361

2. Merli F, Luminari S, Rossi G, Mammi C, Marcheselli L, Ferrari A et al. Outcome of frail elderly patients with diffuse large B-cell lymphoma prospectively identified by Comprehensive Geriatric Assessment: results from a study of the Fondazione Italiana Linfomi. Leukemia Lymphoma. 2014;55(1):38-43. https://doi. org/10.3109/10428194.2013.788176 PMid:23517562

3. Juweid ME. FDG-PET/CT in Lymphoma, in Positron Emission Tomography. Berlin, Germany: Springer; 2011. p. 1-19.

4. Ben-Yakov G, Alao H, Haydek JP, Fryzek N, Cho MH, Hemmati $\mathrm{M}$, et al. Development of hepatic steatosis after chemotherapy for non-hodgkin lymphoma. Hepatol Commun. 2019;3(2):220-6. https://doi.org/10.1002/hep4.1304 PMid:30766960

5. Saadeh S, Younossi ZM, Remer EM, Gramlich T, Ong JP, Hurley $\mathrm{M}$, et al. The utility of radiological imaging in nonalcoholic fatty liver disease. Gastroenterology. 2002;123(3):745-50. https://doi.org/10.1053/gast.2002.35354

PMid:12198701

6. Boellaard R, Delgado-Bolton R, Oyen WJ, Giammarile F, Tatsch K, Eschner W, et al. FDG PET/CT: EANM procedure guidelines for tumour imaging: Version 2.0. Eur J Nucl Med Mol Imaging. 2015;42(2):328-54. https://doi.org/10.1007/s00259-014-2961-x PMid:25452219
7. Salomon T, Nganoa C, Gac AC, Fruchart C, Damaj G, Aide N, et al. Assessment of alteration in liver 18 F-FDG uptake due to steatosis in lymphoma patients and its impact on the Deauville score. Eur J Nucl Med Mol Imaging. 2018;45(6):941-50. https:// doi.org/10.1007/s00259-017-3914-y

PMid:29279943

8. Berthet L, Cochet $\mathrm{A}$, Kanoun $\mathrm{S}$, Berriolo-Riedinger $\mathrm{A}$ Humbert $\mathrm{O}$, Toubeau $\mathrm{M}$, et al. In newly diagnosed diffuse large B-cell lymphoma, determination of bone marrow involvement with 18F-FDG PET/CT provides better diagnostic performance and prognostic stratification than does biopsy. J Nucl Med. 2013;54(8):1244-50. https://doi.org/10.2967/ jnumed.112.114710

PMid:23674577

9. Lin CY, Lin WY, Lin CC, Shih CM, Jeng LB, Kao CH. The negative impact of fatty liver on maximum standarduptake value of liver on FDG PET. Clin Imaging. 2011;35(6):437-41. https:// doi.org/10.1016/j.clinimag.2011.02.005 PMid:22040787

10. Lin $\mathrm{CY}$, Ding $\mathrm{HJ}$, Lin CC, Chen $\mathrm{CC}$, Sun SS, Kao $\mathrm{CH}$ Impact of age on FDG uptake in the liver on PET scan. Clin Imaging. 2010;34(5):348-50. https://doi.org/10.1016/j. clinimag.2009.11.003 PMid:20813297

11. Keramida G, Potts J, Bush J, Verma S, Dizdarevic S, Peters AM. Accumulation of 18F-FDG in the liver in hepatic steatosis. Am J Roentgenol. 2014;203(3):643-8. https://doi.org/10.2214/ AJR.13.12147 PMid:25148170

12. Sarikaya I, Albatineh AN, Sarikaya A. Revisiting weightnormalized SUV and lean-body-mass-normalized SUV in PET studies. J Nucl Med Technol. 2020;48(2):163-7. https://doi. org/10.2967/jnmt.119.233353

PMid:31604893 